\title{
Gaseous outflows in Seyferts and unification: The case of Mrk 533
}

\author{
Prajval Shastri ${ }^{1}$, Jayant Murthy ${ }^{1}$, \\ John Hutchings ${ }^{2}$ and Beverley J. Wills ${ }^{3}$ \\ ${ }^{1}$ Indian Institute of Astrophysics, Bangalore, India email: pshastri@iiap.res.in \\ ${ }^{2}$ Herzberg Institute of Astrophysics, 5071 West Saanich Road, Victoria, BC, V9E 2E7, Canada \\ ${ }^{3}$ Department of Astronomy, University of Texas, Austin TX 78712, USA
}

\begin{abstract}
We present our recent results from the observation of the O VI $\lambda \lambda 1032,1038$ emission doublet in Seyfert galaxies of type 2 with the FUV spectrograph on the FUSE satellite. These observations are part of our investigation to contrast the properties of the OVI emission line and the absorbing outflows in a sample of rigorously matched Seyfert 1s and $2 \mathrm{~s}$, in the framework of the Unified Scheme. The OVI emission line is an excellent diagnostic of the outflowing hot gas at temperatures of $\sim 10^{6} \mathrm{~K}$. In the Unified Scheme, Seyferts of type 2 are those whose central regions are obscured by the ubiquitous dusty torus. We interpret our results in this framework.
\end{abstract}

\section{Introduction}

It is well known that biploar synchrotron-emitting outflowing jets from the nuclei of active galaxies occur not only in the radio-loud AGN, but are also present in the radio-quiet AGN, though with much lower power and sizes. It is also well known that energetic outflows from AGN manifest as line emission from ionized gas, at least some of which is also in bipolar outflow, and often along a direction close to the radio-jet. We have seen many spectacular results from FUSE and Chandra (e.g., Kaspi, and Kriss, these proceedings), wherein Seyferts in particular have outflows with intrinsic absorption in multiple components, implying complex kinematics. The $F U V$ absorption occurs together with warm absorption in X-rays, and is variable (e.g., Kriss, these proceedings.)

Most of these studies, however, and especially those by FUSE, have been done for the brightest AGN, and the bright Seyferts mostly turn out to be those of type 1, viz., those with clearly detectable permitted lines in the optical that are broader than their forbidden lines. Within the framework of the Unified Scheme, which attempts to unify Seyferts of the two types, (e.g., Antonucci 1993) the type 1 objects are those with a direct view of the central regions. On the other hand, little is known about the corresponding properties in Seyfert 2 galaxies, which are those with similar widths of the permitted and forbidden lines, and, in the Unified Scheme, have our line of sight to the central region obscured by an optically thick torus.

Our aim was to compare the hot gaseous outflows in Seyferts of the purportedly poleon and edge-on kinds, as manifested in the Far Ultraviolet OVI emission line and its associated absorption. FUSE (Sahnow et al. 2000) can probe such outflows with unprecedented spectral resolution. In order to rigorously test the Unified Scheme, Seyferts of the two types being compared ought to be selected to be intrinsically similar in the framework of the Scheme. We present results from observations of one of the Seyfert $2 \mathrm{~s}$ from such a sample that we have constructed, viz., Mrk 533 (NGC 7674), which is believed to have a central "hidden" Broad Emission Line Region, detected periscopically 
via spectropolarimetry (Tran 1995). The [OIII] image from HST (Schmitt et al. (2003), Whittle 2004, in preparation) clearly shows extended structure, which is approximately in the direction of the radio jet (Momjian et al. 2003).

\section{The FUSE data}

Using FUSE, we observed Mrk 533 through a $30^{\prime \prime}$ aperture with an effective area near the OVI lines of $26 \mathrm{~cm}^{2}$, and a total exposure of $\approx 20 \mathrm{ksecs}$. Pre-processed data from the FUSE pipeline were used for the reductions.

\section{Results}

We clearly detect OVI $\lambda \lambda 1031,1037$ emission in both the LiF1A and LiF2B detectors at the expected cosmologically redshifted position $(\mathrm{z}=0.0289)$, with the doublet clearly separated ( $c f$. , Fig. 1). We note that the night-time only data are consistent with the total data except for the terrestrial airglow lines, as expected. The data from the two different exposures are also consistent, including some of the prominent dips.

It is well-known that Galactic molecular hydrogen causes a very large number of contaminating absorptions in AGN spectra from FUSE. These absorptions have to be accounted for by modelling the hydrogen spectrum. The model spectrum of molecular Hydrogen, with no redshift, is plotted in the top panel of the figure. It is seen that some of the dips in the spectrum of Mrk 533 can consistently be accounted for by the stronger dips in the model spectrum.

In the middle panel of the figure, the same data as above, summed for the two different detectors are plotted in velocity space. Data for the two emission lines are summed and plotted below in the figure. The dips in the spectrum, that are shaded grey, are those which are consistent with all the stronger dips in the model $H_{2}$ spectrum.

It is clear that even after accounting for the Galactic absorption, the net OVI profile still shows a blue wing. Also, there are residual dips (shaded black in Fig. 1), which are not attributable to Galactic hydrogen. We interpret these dips as intrinsic to Mrk 533, corresponding to velocities of -200 and $-800 \mathrm{~km} \mathrm{~s}^{-1}$ respectively.

We also detect the CIII line at $977 \AA$ (Fig. 1, bottom panel). Although the data are rather noisy, it is intriguing that a clear dip is seen in both detectors, at $\approx-200 \mathrm{~km} \mathrm{~s}^{-1}$. Both because this dip occurs in a region clear of Galactic $\mathrm{H} 2$ absorption, and because it is consistently seen in both the detectors, we interpret it as due to intrinsic absorption in Mrk 533. It is interesting that this absorption occurs at a velocity where an intrinsic absorption component is seen in the OVI doublet also.

\section{Discussion}

The OVI line (a permitted line) of Mrk 533 is "narrow" in the sense of having a FWHM that is $<1000 \mathrm{~km} \mathrm{~s}^{-1}$ and similar to the forbidden lines. This is in predicted contrast to the OVI line seen in purportedly pole-on Seyferts (type 1s) which show a broad component, sometimes accompanied by a narrower one (e.g., Kriss, these proceedings.). The intensity ratio of $\approx 1$ for the doublet is indicative of optically thick emission.

A strong blue-wing in several emission lines of Mrk533 has been known for a long time - e.g. Veilleux (1991) has documented that the blue wing of the [OIII] $\lambda \lambda 4959,5007$ line stretches out to a spectacular $2000 \mathrm{~km} \mathrm{~s}^{-1}$. The STIS image in the [OIII]5007 region (Whittle 2004, in preparation) confirms this. A standard interpretation of the blue wing has been a combination of outflow and obscuration, and the torus model required by 

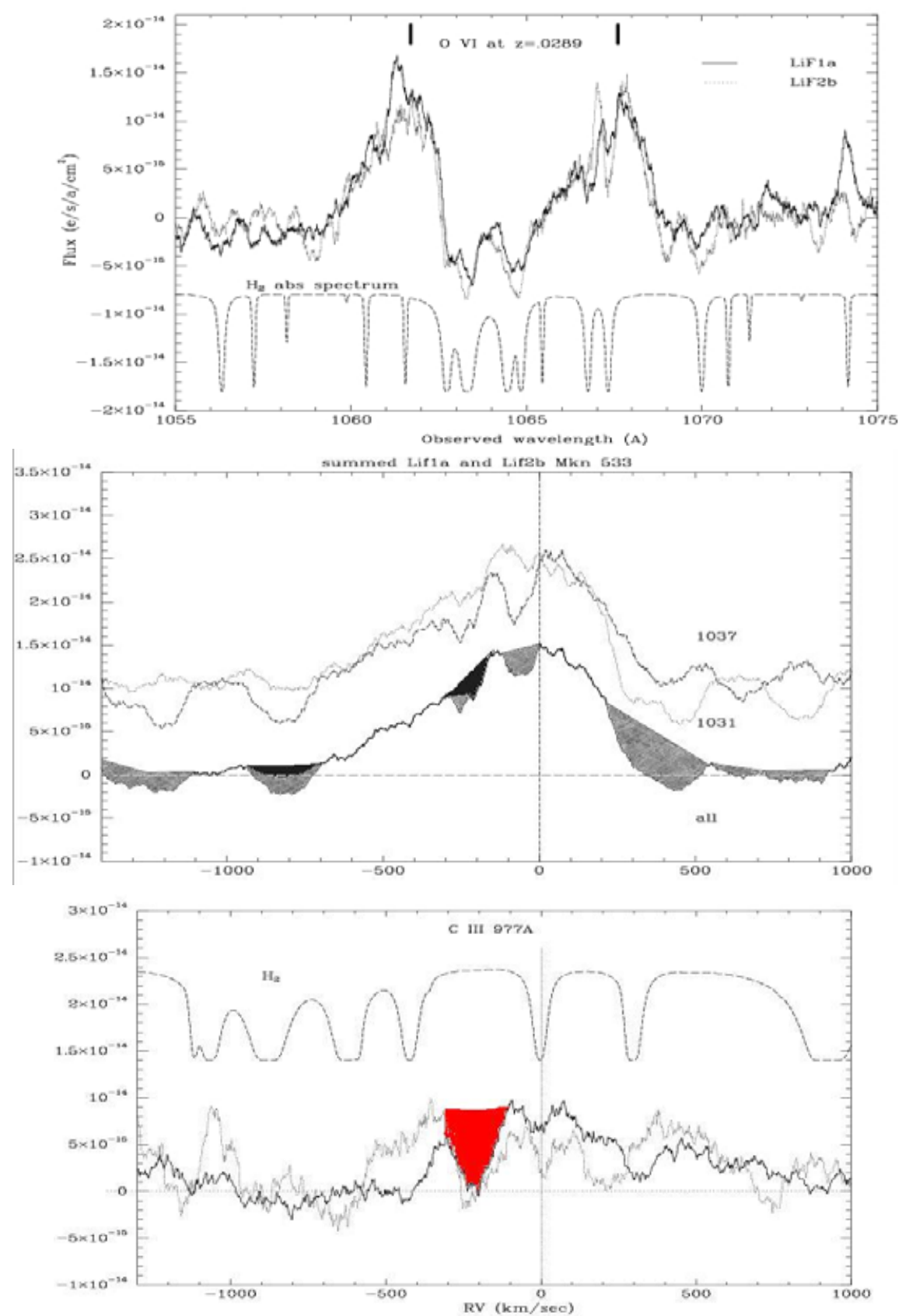

Figure 1. Top panel: The spectrum of Mrk 533 in the redshifted OVI doublet region. Data are from the FUSE detectors LiF1a and LiF2b, which give redundant coverage of this region. Below is plotted the model absorption spectrum of Galactic $\mathrm{H}_{2}$, illustrating that several of the dips in the Mrk 533 spectra are due to contamination from Galactic absorption. Middle panel: The same spectra in velocity space. At the bottom is the combination of all the four spectra showing both the Galactic contamination (shaded grey) and the intrinsic dips (black). Bottom panel: The spectrum of Mrk 533 in the redshifted CIII line region, plotted in velocity space. The CIII line is detected, but with relatively low signal-to-noise. Above it is plotted the corresponding model absorption spectrum of Galactic $\mathrm{H}_{2}$. We interpret the dip at $\sim-200 \mathrm{~km} \mathrm{~s}^{-1}$ (shaded black) to be intrinsic to Mrk 533, since it occurs in the spectra from both detectors and in a region clean of Galactic absorption. 
unification can produce such an effect. In the case of Mrk 533, it is clear from the spatial distribution of the blue wing in the STIS image that the wing is from an approaching outflow.

Other optical emission lines $(\mathrm{H} \alpha,[\mathrm{NII}])$ also known to show a blue wing (Veilleux 1991), though with a lower degree of asymmetry. In fact, de Robertis \& Shaw (1990) found, for several Seyferts, including MRK 533, a correlation between the asymmetry of the emission lines in a given object and their ionization potential. This had the obvious interpretation that the higher excitation lines originate closer to the ionizing continuum, i.e., the nucleus, and therefore also come from the fastest material. However the OVI line that we observe, though of extreme excitation, is less asymmetric than the [OIII] line, and thus is not consistent with this correlation extending to higher ionization potentials.

The CIII emission is only weakly detected, and therefore no meaningful analysis of its profile characteristics can be done. It is intriguing, however, that the absorption trough at $\approx-200 \mathrm{~km} \mathrm{~s}^{-1}$ which is most likely to be intrinisic absorption at the same velocity relative to systemic as one of the troughs in the OVI line profile.

The observation of intrinsic absorption troughs would be consistent with an accelerated wind from the central source, with the the blue-shifted absorption being from a cloud in the outer region of the conical outflow (e.g., Hutchings et al. 2001; de Kool 1997).

\section{Acknowledgements}

We thank Suzy Collin for several insightful discussions, and Mark Whittle for his wonderful spectral images from a paper in preparation that were presented at the conference. PS acknowledges travel grants from the IAU and the Indian National Science Academy, and the tremendous help from the organisers that enabled her participation in the conference.

\section{References}

Antonucci, R. 1993, ARA\&A, 31, 473

de Robertis, M. M. \& Shaw, R. A. 1990, ApJ, 348, 421

Momjian, E., Romney, J. D., Carilli, C. L., \& Troland, T. H. 2003, ApJ, 597, 809

Sahnow, D. J., Moos, H. W., Friedman, S. D., et al. 2000, in Proc. SPIE Vol. 4139, p. 131136, Instrumentation for UV/EUV Astronomy and Solar Missions (eds. S. Fineschi; C. M. Korendyke; O. H. Siegmund; B. E. Woodgate), 131

Schmitt, H. R., Donley, J. L., Antonucci, R. R. J., Hutchings, J. B., \& Kinney, A. L. 2003, ApJS, 148, 327

Tran, H. D. 1995, ApJ, 440, 578

Veilleux, S. 1991, ApJ, 369, 331 